\title{
Estudio de la resistencia mecánica a la compresión de ladrillos elaborados a partir de mezclas arcilla roja-cemento Portland
}

\author{
José López-Muñoz, Lester Espinosa-Pérez, Rolando Guevara-Arróliga* \\ Facultad de Ingeniería Química, Universidad Nacional de Ingeniería (UNI) \\ Avenida Universitaria, PO Box 5595, Managua, Nicaragua \\ (recibido/received: 30-Oct-2014; aceptado/accepted: 19-Dic-2014)
}

\begin{abstract}
RESUMEN
Se presenta una propuesta para la elaboración de ladrillos artesanales a base del suelo arcilloso estabilizado con cemento Portland gris, como alternativa para reducir el grado de contaminación causado por emisiones de dióxido de carbono a causa del uso de madera, usada como fuente de combustión durante la etapa de cocción de los ladrillos elaborados artesanalmente a nivel nacional. Se trabajó básicamente con el banco de suelo de una de las ladrilleras ubicada en La Paz Centro, Nicaragua y dos marcas diferentes de cemento portland. Se elaboraron muestras de cemento/suelo según las normas y se compararon las propiedades físico mecánicas con los ladrillos cocidos por medio de un diseño de experimento de tipo factorial mixto completo. Los factores que se estudiaron fueron las porciones de cemento/suelo, agua/ligante y marca de cemento. Los mejores resultados para muestras de cemento/suelo presentaron resistencias superiores a los $12 \mathrm{MPa}$ después de 28 días de curado y porcentajes de absorción inferiores al 15\%. Los productos de hidratación responsables del desarrollo de la resistencia mecánica se analizaron por medio de pruebas de espectroscopia infrarroja, mostrando la mayor cantidad de silicatos de calcio hidratado en muestras con cemento Canal.
\end{abstract}

Palabras clave: Resistencia mecánica a la compresión; Absorción; Peso unitario; Espectro; Silicatos de calcio hidratados

\begin{abstract}
A proposal for the development of handcrafted bricks of clay soil stabilized with gray portland cement, as an alternative to reduce the level of pollution caused by emissions of carbon dioxide from the use of wood as a source of combustion during the baking step of bricks is presented. The work was carried out in the soil bank of one brickyard located in La Paz Centro, Nicaragua. Moreover, two different brands of Portland cement were used. Cement/soil samples were prepared according to the established norms, and the mechanical-physical properties were compared with bricks cooked through a full mixed factorial design experiment. The parameters studied were ratios of cement/soil, water/cement binder and cement brand. The best results for samples of cement/soil reached resistances higher than $12 \mathrm{MPa}$ after 28 days of curing and absorption percentages lower than $15 \%$. Hydration products, responsible for the development of mechanical strength, were analyzed using infrared spectroscopy. The largest number of hydrated calcium silicate was obtained for samples with Canal cement.
\end{abstract}

Keywords: Absorption; Hydrated calcium silicates; Unitary weight; Spectrum; Compressive mechanical resistance

\footnotetext{
*Autor para la correspondencia. Tel.: +505 22781463.

Correo electrónico: rolando.guevara@fiq.uni.edu.ni (R. Guevara).
} 


\section{Introducción}

Los avances tecnológicos han ayudado mucho en la mejora de los materiales de construcción, buscando diversas formas de cómo resistir las adversidades de la naturaleza y a la vez proteger al medio ambiente de la contaminación.

Algunos de los materiales más utilizados en la construcción son el cemento Portland, la arcilla y la arenilla, los cuales son muy importantes en la elaboración de ladrillos cocidos.

Actualmente en Nicaragua existen diversas fábricas artesanales, las cuales elaboran ladrillos para la construcción. Muchas de estas industrias principalmente las artesanales, utilizan un proceso de cocción en la elaboración de éstos ladrillos, la cual se le conoce como quemado. En éste se utilizan como fuente de combustible: leña y el aserrín, entre otros materiales como llantas y carbón, que puedan generar una temperatura cercana a los $900{ }^{\circ} \mathrm{C}$, la cual ocasiona un aumento en la densidad y mejora la resistencia mecánica de los ladrillos. Sin embargo, se incurre en un elevado consumo energético y un alto costo ambiental para poder obtener un ladrillo con los requerimientos básicos de calidad.

El perjuicio al medio ambiente por emisiones de gases invernaderos como el dióxido de carbono, que se producen por la combustión de la madera y de otros aditivos, se combina con el despale ocasionado por las industrias que requieren de los recursos de la madera para éste fin y aunque se han hecho esfuerzos para reducir el uso de la madera, como por ejemplo, la incorporación de ventiladores que aumentan la eficiencia de la combustión dentro del horno, mejorando el proceso de quemado de los ladrillos, éstos no son suficientes para reducir el problema.

Es por esto que ésta investigación busca otra alternativa más amigable con el ambiente, utilizando ladrillos basados en suelo-cemento, que a diferencia de los ladrillos de barro frescos, constituyen un material crudo estable y compacto, utilizando la tierra no fértil como materia prima, que es estabilizada con la adición de cemento.
Al suelo que se utilizó para elaborar la mezcla se le realizaron pruebas de granulometría y composición, con el propósito de obtener una mejora en las propiedades físico-mecánicas de los ladrillos, variando las relaciones de proporción entre la arcilla y el cemento en el proceso de fabricación. Todo esto con el objetivo de observar experimentalmente como varía la resistencia mecánica a la compresión en cada composición.

Así, se pretende disminuir notablemente el uso de la madera como combustible, en cada una de las industrias artesanales que elaboran ladrillos en nuestro país. Esta investigación contribuirá no solo a reducir el uso de la madera, sino que también se pretende optimizar el proceso de elaboración de ladrillos obteniendo una mejora en las propiedades físico-mecánicas de su materia prima.

Cabe mencionar que actualmente la entidad reguladora de estos materiales en Nicaragua es el Ministerio de Transporte e Infraestructura (MTI), el cual no ha establecido una norma clara para el control de la elaboración de ladrillos de arcilla roja, por lo que este estudio serviría como base para investigaciones posteriores.

\section{Material y Métodos}

\subsection{Materiales}

El suelo-cemento es una mezcla íntima de suelo, convenientemente pulverizado, con determinadas porciones de agua y cemento que se compacta y cura para obtener mayor densidad. Cuando el cemento se hidrata la mezcla se transforma en un material sólido, durable y rígido. Se le usa principalmente como base en los pavimentos de carreteras, calles y aeropuertos (Besoain, 1985).

En otras palabras, podemos decir que la mezcla de suelo-cemento no es más que la mezcla de suelo no fértil con una porción de cemento y agua adicionados en proporciones adecuadas y compactadas, esta mezcla supera las propiedades de los minerales que posee el suelo como la arcilla y la arenilla, pues su comportamiento físico-mecánico mejora a través de la 
estabilización con aglomerantes minerales como el cemento.

Actualmente en Nicaragua existen dos grandes productoras de cemento que son CEMEX y Holcim S.A. Ambas fábricas producen cemento gris de tipo Portland GU (General Use, por sus siglas en inglés), también producen cemento hidráulico. Generalmente y de acuerdo con estudios previos ya realizados a cerca de las mezclas de suelo-cemento, es recomendable utilizar cemento de tipo Portland, el cual, al ser mezclado con el suelo adquiere propiedades estabilizantes, las cuales una vez hecha la mezcla aumenta la resistencia mecánica.

Cuando el cemento Portland es mezclado con el agua, se obtiene un producto de características plásticas con propiedades adherentes que solidifica en algunas horas $y$ endurece progresivamente durante un período de varias semanas hasta adquirir su resistencia característica (Corneado, 2010). El cemento está compuesto por cuatro componentes principales que son: $67 \% \mathrm{CaO}, 22 \% \mathrm{SiO}_{2}, 5 \% \mathrm{Al}_{2} \mathrm{O}_{3}, 3 \%$ de $\mathrm{Fe}_{2} \mathrm{O}_{3}$, y un $3 \%$ de otros compuestos. Estos componentes están presentes en cuatro minerales esenciales que componen el cemento los cuales son: Alita $\left(\mathrm{Ca}_{3} \mathrm{SiO}_{5}\right)$ que compone entre el 50\%$70 \%$ del cemento Portland, la Belita $\left(\mathrm{Ca}_{2} \mathrm{SiO}_{4}\right)$ constituye entre el 15\%-30\% del cemento Portland, el Aluminato $\left(\mathrm{Ca}_{3} \mathrm{Al}_{2} \mathrm{O}_{6}\right)$ compone entre el 5\%-10\% del cemento y la Ferrita $\left(\mathrm{Ca}_{2} \mathrm{AlFeO}_{5}\right)$ que esta entre el $5 \%$ y el $15 \%$.

\subsection{Ensayo de resistencia mecánica}

El ensayo de resistencia mecánica a la compresión (RMC) de suelo-cemento es de gran importancia en el ámbito de las construcciones de obras, específicamente en las pruebas de control de calidad a la mezcla utilizada en la estabilización de los suelos. El método de ensayo permite conocer las características mecánicas de la mezcla, lo cual le sirve al diseñador para verificar el cumplimiento del resultado esperado en la utilización de dicha mezcla (Amaya y Díaz, 2011).

Los ensayos para determinar la resistencia mecánica en cada ladrillo de suelo-cemento no poseen actualmente una norma que regulen su venta y distribución en el mercado, sin embargo hay normas establecidas por la RCN-07-55 (Reglamento Nacional de la Construcción de Nicaragua) que se utilizan con referencia para esta investigación. El cálculo que se empleará para determinar la fuerza de compresión en cada una de las muestras está dado por la siguiente Ecuación (1):

$$
C=\frac{w}{A}
$$

El porcentaje de absorción se realiza con el propósito de determinar la cantidad o el porcentaje de agua contenida en una unidad de mampostería, en este caso en ladrillos de suelocemento, la cual se determina por medio de las condiciones de saturación y condiciones secas. El cálculo para determinar el porcentaje de absorción en la muestra está determinado por la siguiente Ecuación (2):

$\%$ Absorción $=\frac{W_{s}-W_{d}}{W_{d}} \times 100$

\subsection{Espectroscopía por transformadas de Fourier}

El uso de la espectroscopía infrarroja por transformadas de Fourier (FTIR por sus siglas en Inglés) es muy útil para brindar información de materiales desconocidos, determinar la calidad y consistencia de una muestra, determinar la cantidad de componentes en una mezcla entre otras aplicaciones más, sin embargo para este estudio se utilizó esta técnica con el objetivo de brindarnos información un poco más detallada de los grupos funcionales que se encuentran presentes en las muestras de suelo-cemento como producto de las reacciones de hidratación entre el cemento y la arcilla.

\subsection{Diseño experimental}

El diseño de experimento que se realizó para esta investigación es de tipo factorial mixto completo, para el cual se tienen dos factores a tres niveles $\left(3^{2}\right)$, un factor a dos niveles $\left(2^{1}\right)$ y tres variables verdad o respuesta los cuales son: 
la RMC, el \%Absorción y el peso unitario. Las relaciones que se tomaron para el diseño de experimento se detallan en la Tabla 1.

Tabla 1 Factores y niveles del primer diseño experimental.

\begin{tabular}{lccc}
\hline \multicolumn{1}{c}{ Factores } & \multicolumn{3}{c}{ Niveles } \\
\hline C/S: relación & $(\mathrm{C} / \mathrm{S})_{1}:$ & $(\mathrm{C} / \mathrm{S})_{2}:$ & $(\mathrm{C} / \mathrm{S})_{3}:$ \\
cemento/suelo & $1 / 4$ & $1 / 7$ & $1 / 10$ \\
$\mathrm{Ag} / \mathrm{L}:$ relación & $(\mathrm{Ag} / \mathrm{C})_{1}:$ & $(\mathrm{Ag} / \mathrm{C})_{2}:$ & $(\mathrm{Ag} / \mathrm{C})_{3}:$ \\
agua/ligante & 0.15 & 0.175 & 0.2 \\
Mc: marca de & $\mathrm{Mc}_{1}:$ Canal $\quad \mathrm{Mc}_{2}:$ Holcim \\
cemento & \multicolumn{4}{c}{} \\
\hline
\end{tabular}

El diseño de experimento fue diseñado con los datos de la Tabla 1, utilizando el software Minitab 16 V. Español. El programa permite elegir el tipo de diseño experimental que será utilizado tomando en consideración la cantidad de factores o variables que serán estudiados, además de ingresar la cantidad de niveles por cada factor. En la Tabla 2 se muestra la combinación de los factores por cada nivel, el cual originó cada una de los tratamientos experimentales que se realizó de acuerdo a la respuesta del software.

El procedimiento que se empleó para la elaboración de la mezcla y los especímenes se realizó con base en la norma ASTM C109/C 109M-08, sin embargo se realizaron algunas modificaciones al procedimiento ya que se tomó en cuenta parte del desarrollo metodológico que plantea Gatani (2000) en su investigación acerca de ladrillos de suelo-cemento.

La realización de ésta prueba se hizo de forma adicional al estudio de esta investigación ya que aporta información muy valiosa y de carácter científico, la cual se llevó a cabo gracias a la colaboración del Laboratorio de materiales y modelos estructurales (LanammeUCR) de la Universidad de Costa Rica.

Para aportar otros resultados a éste estudio se decidió escoger seis muestras o especímenes cúbicos, una de ellas con los mejores resultados obtenidos en las pruebas de resistencia del primer diseño factorial, tres muestras elaboradas con tratamiento térmico a $700{ }^{\circ} \mathrm{C}, 800{ }^{\circ} \mathrm{C}$ y 900 ${ }^{\circ} \mathrm{C}$, las dos últimas muestras se tomaron sin tratamiento térmico pero con diferente marca de cemento utilizada.

Tabla 2 Diseño Factorial para la mezcla de suelo-cemento.

\begin{tabular}{cccc}
\hline No. Exp. & $\mathrm{Ag} / \mathrm{L}$ & $\mathrm{C} / \mathrm{S}$ & $\mathrm{Mc}$ \\
\hline 1 & 0.15 & $1 / 7$ & Holcim \\
2 & 0.2 & $1 / 7$ & Canal \\
3 & 0.175 & $1 / 4$ & Holcim \\
4 & 0.15 & $1 / 4$ & Canal \\
5 & 0.15 & $1 / 7$ & Canal \\
6 & 0.15 & $1 / 10$ & Holcim \\
7 & 0.2 & $1 / 10$ & Holcim \\
8 & 0.2 & $1 / 10$ & Canal \\
9 & 0.15 & $1 / 10$ & Canal \\
& & & \\
No. Exp. & $\mathrm{Ag} / \mathrm{L}$ & $\mathrm{C} / \mathrm{S}$ & Mc \\
10 & 0.15 & $1 / 10$ & Canal \\
11 & 0.2 & $1 / 7$ & Holcim \\
12 & 0.15 & $1 / 4$ & Holcim \\
13 & 0.175 & $1 / 7$ & Holcim \\
14 & 0.175 & $1 / 7$ & Canal \\
15 & 0.175 & $1 / 4$ & Canal \\
16 & 0.175 & $1 / 10$ & Canal \\
17 & 0.175 & $1 / 10$ & Holcim \\
18 & 0.2 & $1 / 4$ & Holcim \\
\hline
\end{tabular}

Ag/L: Relación agua-ligante

C/S: Relación de cemento y suelo en peso

Mc: Marca de cemento

\section{Resultados y Discusión}

En los ensayos preliminares se realizaron pruebas de RMC a los ladrillos de arcilla roja que se elaboraron en la ladrillera Bella Vista. Dentro de estos ensayos se realizaron pruebas de dos tipos, en la primera se midió la RMC al ladrillo completo y en la segunda prueba se cortaron cubos de los ladrillos completos con dimensiones aproximadas a los especímenes que se elaboraron en el laboratorio con el fin de realizar una mejor comparación de la resistencia mecánica.

Para los ensayos de RMC de los ladrillos cocidos se tomaron seis unidades de tamaño estándar y de mayor demanda según el propietario de la ladrillera, de los cuales se escogieron cuatro al azar y se ensayaron, los dos restantes se cortaron en cubos obteniendo un área superficial de 5 pulgada $^{2}$ con una diferencia de una pulgada cuadrada de los especímenes 
elaborados en el laboratorio. En la Tabla 3 se muestran los resultados de RMC para los dos tipos de pruebas.

Tabla 3 RMC de ladrillos cocidos.

\begin{tabular}{cccc}
\hline \multicolumn{2}{c}{ Cubos } & \multicolumn{2}{c}{ Ladrillos } \\
\hline Peso & RMC & Peso & RMC \\
$(\mathrm{g})$ & $(\mathrm{Mpa})$ & $(\mathrm{g})$ & $(\mathrm{Mpa})$ \\
218.15 & 2.48 & 4528.00 & 8.90 \\
193.19 & 3.06 & 4614.00 & 7.22 \\
191.54 & 3.03 & 4370.00 & 11.77 \\
201.06 & 3.86 & 4492.00 & 9.50 \\
Promedio: & 3.11 & Promedio: & 9.35 \\
\hline
\end{tabular}

Como se aprecia en la tabla anterior la resistencia mecánica promedio de los ladrillos cortados en forma de cubos tienen un promedio de $3.11 \mathrm{MPa}$ y una resistencia promedio de 9.35 $\mathrm{MPa}$ como ladrillo completo. La baja resistencia que se obtuvo de estos resultados se logró apreciar por la cantidad de poros que tenía el material ya que al someter el ladrillo al horno para su cocimiento a una alta temperatura la cantidad de agua que contiene el ladrillo se evapora rápidamente dejando grandes y pequeños poros dentro del ladrillo lo que ocasiona su bajo rendimiento en la resistencia mecánica y aumentando su porcentaje de absorción de agua.

En la Fig. 1 se tienen los resultados de RMC promedio obtenidos de los experimentos ensayados para la relación $1 / 10$, es decir para una parte de cemento por diez de suelo variando en cada gráfica la relación de agua-ligante.

Los resultados que se presentan en la Fig. 1 son parte de los ensayos de resistencia mecánica que se realizaron a diferentes edades de curado de los especímenes, en los cuales se puede apreciar que en las Figs. $7.4 \mathrm{a}$ y $7.4 \mathrm{~b}$, la curva de experimentos realizada con cemento Holcim está por encima de la curva del cemento Canal obteniendo de esta manera resistencias superiores a los $5 \mathrm{MPa}$, por otro lado se observó que después de los 14 días de curado la curva de cemento Holcim continúa su evolución normal de RMC, lo cual pudo haber ocurrido porque algunos de los especímenes que se elaboraron no llevaron a cabo una reacción de hidratación completa.

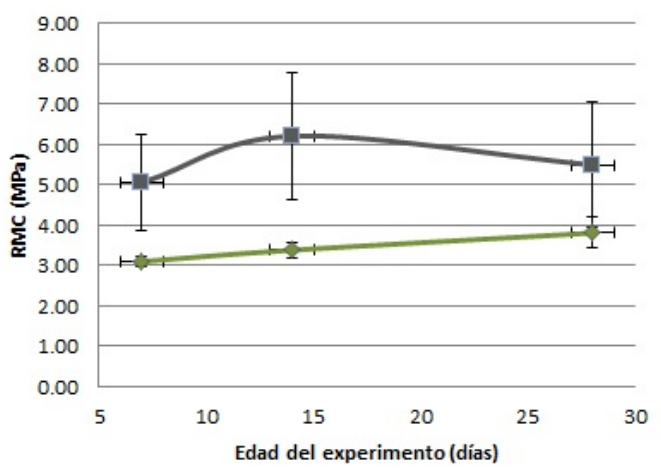

a)

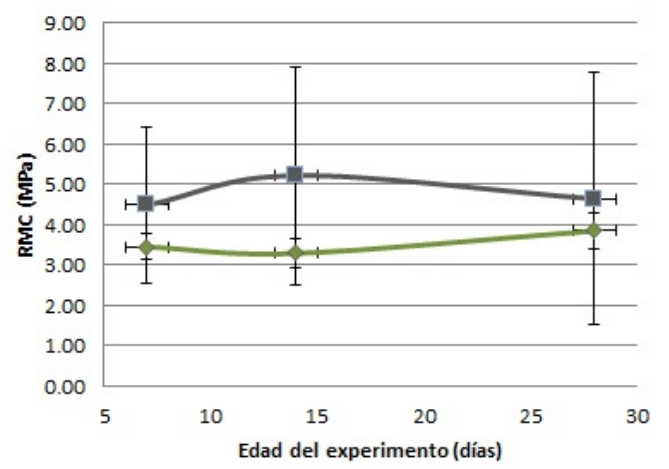

b)

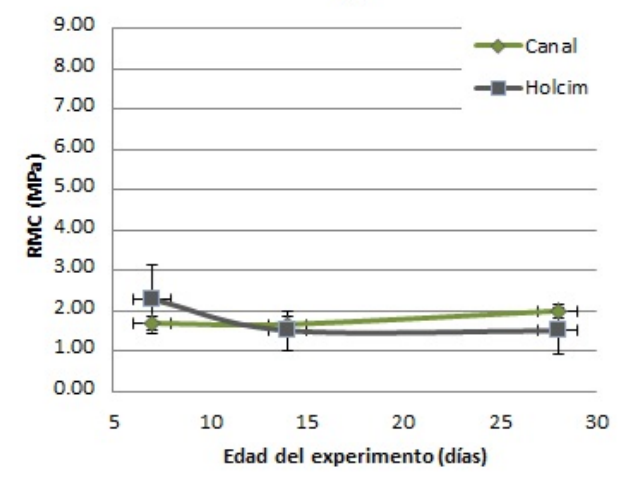

c)

Fig. 1 Resultados de RMC para relaciones de C/S de 1/10. a) Con 0.2 de Ag/L. b) Con 0.175 de $\mathrm{Ag} / \mathrm{L}$. c) Con 0.15 de $\mathrm{Ag} / \mathrm{L}$.

En los resultados que se obtuvieron para las relaciones de $1 / 7$, es decir una parte de cemento por siete de suelo como se muestra en la Fig. 2 se observa un comportamiento similar en el cual la curva de cemento Holcim permanece por 
encima de la otra curva, con la diferencia de la relación anterior en que el grado de separación de ambas curvas es mucho menor, dicho comportamiento es el resultado del incremento de la porción de cemento que se le adiciona a la mezcla, ya que al haber un mayor número de partículas de cemento hay una mayor interacción con la actividad puzolanica de la arcilla, además en referencia a los resultados de RMC obtenidos hay un pequeño incremento de casi un (1) MPa en cada experimento.

Los mejores resultados de RMC que se obtuvieron para las relaciones más bajas de cemento/suelo, es decir de 1/4 y para las relaciones más altas de agua/ligante de 0.2 , sin embargo para las demás relaciones de 0.175 y 0.15 también se tuvieron incrementos de resistencia como se observa en la Fig. 3. A diferencia de las figuras anteriores se observó que hay un mejor rendimiento del cemento Canal cuando las relaciones en peso de cemento y suelo son las más bajas y con adiciones grandes de agua, lo cual se puede apreciar en las curvas donde la curva de cemento Canal pasó a estar por encima de la curva de cemento Holcim llegando a una resistencia máxima de 12.57 $\mathrm{MPa}$.

Realizando un análisis de los datos promedio obtenidos con los resultados de las pruebas de RMC a los 28 días de curado, se utilizó el software Minitab v.16 con el cual se obtuvo un gráfico de interacciones y un gráfico de efectos principales entre las tres variables de estudio, es decir la relación de cemento/suelo, agua/ligante y la marca de cemento, en los cuales se observa más detalladamente la interacción y el efecto entre las tres variables.

En la Fig. 4 se puede apreciar la interacción entre las variables, en el cual se observa que la curva de color verde es la que tiene los mejores resultados tanto para la relación de $\mathrm{C} / \mathrm{S}$ como para la relación de $\mathrm{Ag} / \mathrm{L}$. La interpretación de la gráfica se basa en la dirección y crecimiento de las curvas, donde un aumento en la relación de agua sea indiferente la relación de $\mathrm{C} / \mathrm{S}$ se tendrá un crecimiento proporcional en la resistencia mecánica y se tienen mejores resultados utilizando el cemento Canal.

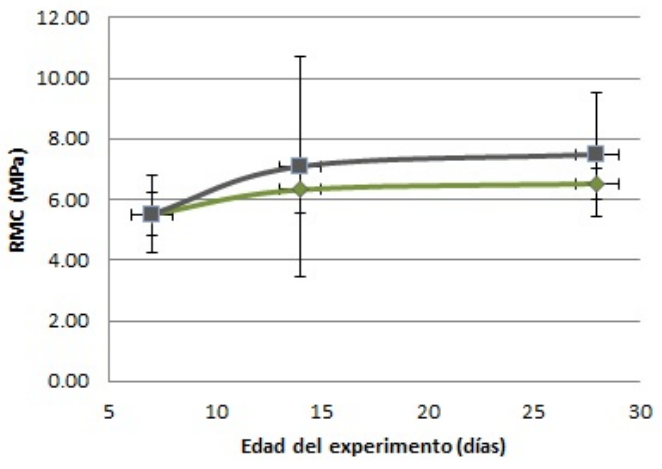

a)

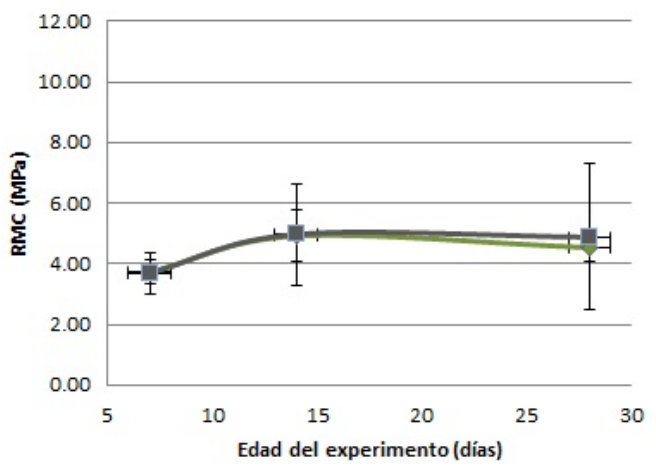

b)

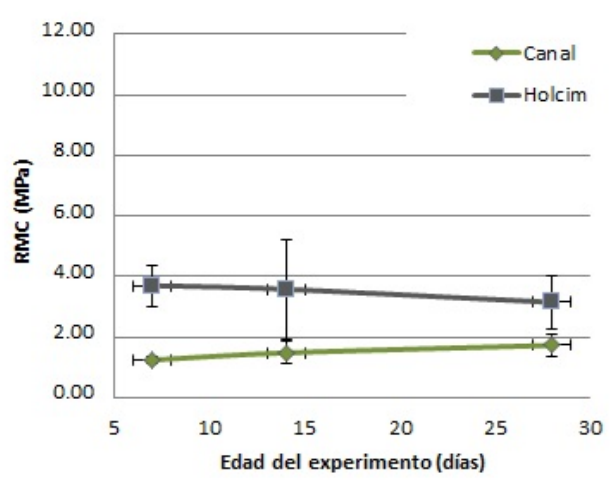

c)

Fig. 2 Resultados de RMC para relaciones de C/S de 1/7. a) Con 0.2 de Ag/L. b) Con 0.175 de $\mathrm{Ag} / \mathrm{L}$. c) Con 0.15 de Ag/L.

La Fig. 5 muestra que de efectos principales toma como base todos los resultados obtenidos de las pruebas de RMC a los 28 días de curado del cual se observa el efecto de los datos en correspondencia a la línea central del gráfico que representa la media estadística de los datos. 


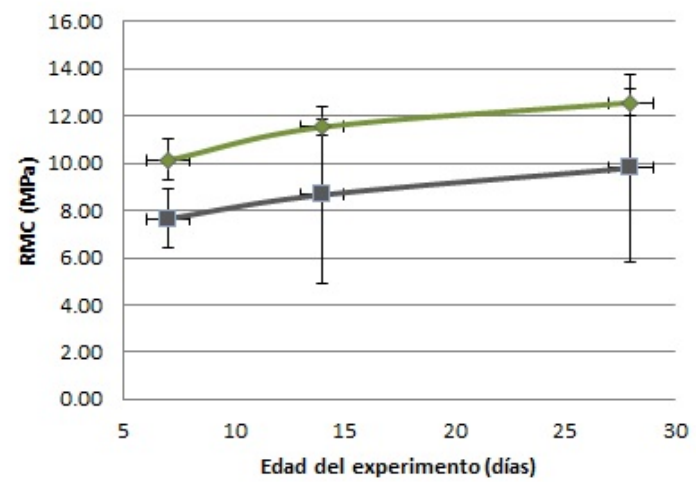

a)

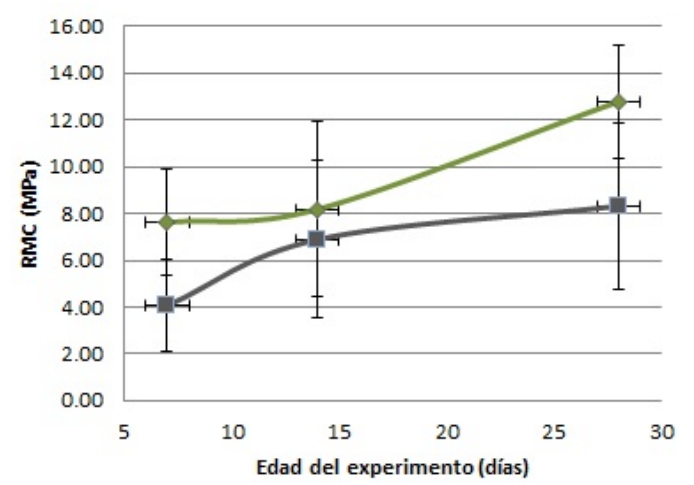

b)

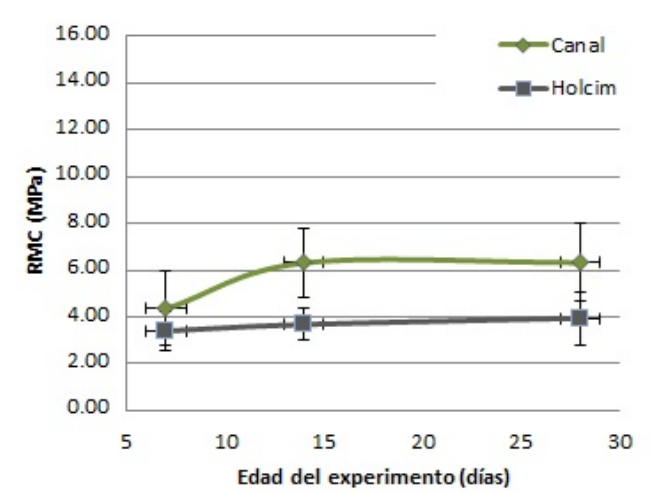

c)

Fig. 3 Resultados de RMC para relaciones de C/S de 1/4. a) Con 0.2 de Ag/L. b) Con 0.175 de $\mathrm{Ag} / \mathrm{L}$. c) Con 0.15 de Ag/L.

En la Fig. 5 se puede observar que para las relaciones de $\mathrm{C} / \mathrm{S}$ de $1 / 10$ y $1 / 7$ se encuentran por debajo de la media, es decir que los resultados obtenidos para estas proporciones son menores a los $6 \mathrm{MPa}$, lo cual indica que para una proporción de $80 \%$ de suelo y $20 \%$ de cemento
Portland se obtienen resistencias más altas, así mismo en las relaciones de $\mathrm{Ag} / \mathrm{L}$ se puede ver que entre más grande sea la relación de agua que se le adicione a la mezcla de morteros se incrementará la RMC, además se aprecia que con relaciones superiores a 0.175 los resultados de resistencia estarán por encima de los $6 \mathrm{MPa}$. En relación a la marca de cemento que se utilizó se nota que para cementos de tipo Portland elaborados por Cemex se tienen resultados superiores a la media, sin embargo la diferencia es pequeña en comparación con los resultados que se obtuvieron con el cemento Holcim.

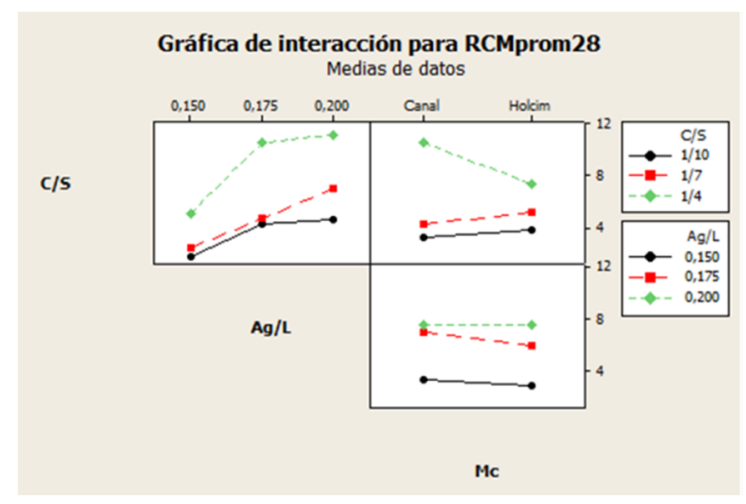

Fig. 4 Gráfica de interacción para RMC promedio.

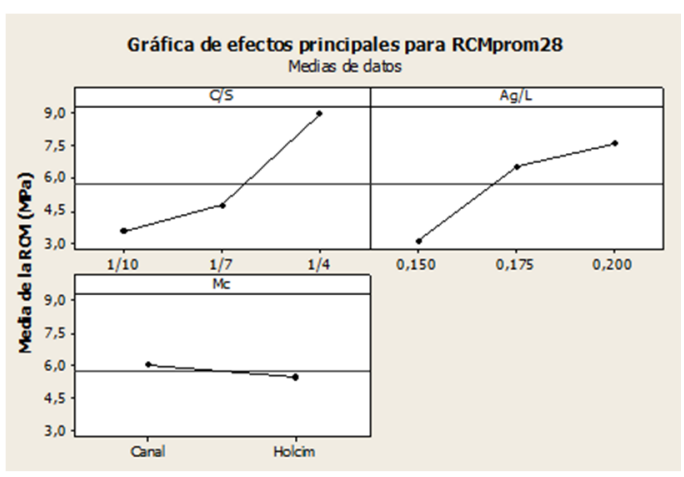

Fig. 5 Gráfica de efectos principales de RMC promedio.

Otro de los análisis que se le realizaron a los datos recopilados de los resultados de RMC, se hizo con la gráfica de valores individuales, en el cual se detalla cada una de las mediciones realizadas por experimento con un intervalo de confianza del $95 \%$ para la media como se indica en la Fig. 6. 


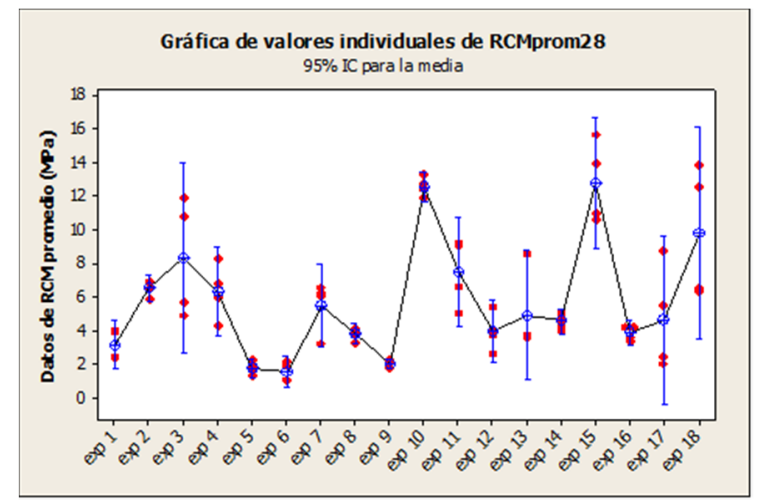

Fig. 6 Gráfica de valores individuales de RMC promedio.

En la Fig. 6 se puede observar que la línea azul en cada uno de los puntos de los experimentos representa la desviación estándar de los datos, es decir los límites superiores e inferiores que corresponden al margen de error, además se puede apreciar que un pequeño círculo azul representa la media de los cuatro ensayos realizados por cada uno de los experimentos. Este tipo de gráfica muestra a simple vista que tan grande es la desviación de los datos y que tan precisos has sido los resultados de los ensayos en correspondencia con la media, ya que entre más cerca estén los puntos de los ensayos realizados a la media más preciso es el resultado. De los 18 experimentos realizados se puede apreciar del gráfico que el experimento 10 obtuvo la más alta resistencia así como la menor desviación estándar.

Con respecto al porcentaje de absorción éste ensayo también se relaciona mucho con la resistencia mecánica, ya que esta prueba depende del grado de compactación de las muestras, el tamaño y distribución de las partículas de lo cual dependerá el tamaño de los poros que se formen dentro de los compósitos elaborados de suelo-cemento.

En la Fig. 7 se muestra la comparación del porcentaje de absorción de cinco muestras de ladrillos cocidos y cinco muestras de morteros de suelo-cemento con los mejores resultados de RMC. Ladrillos cocidos tienen un porcentaje más alto de absorción de agua lo cual se debe a la cantidad de poros de distintos tamaños que tienen en su estructura, formados debido a que la mezcla que se elabora en la ladrillería artesanal contiene un porcentaje muy grande de agua con el propósito de ser moldeado fácilmente. El porcentaje de agua promedio alcanzado por los ladrillos cocidos alcanza casi el $24 \%$ a diferencia de las muestras de suelo-cemento que no superan el $15 \%$ para las mejores relaciones.

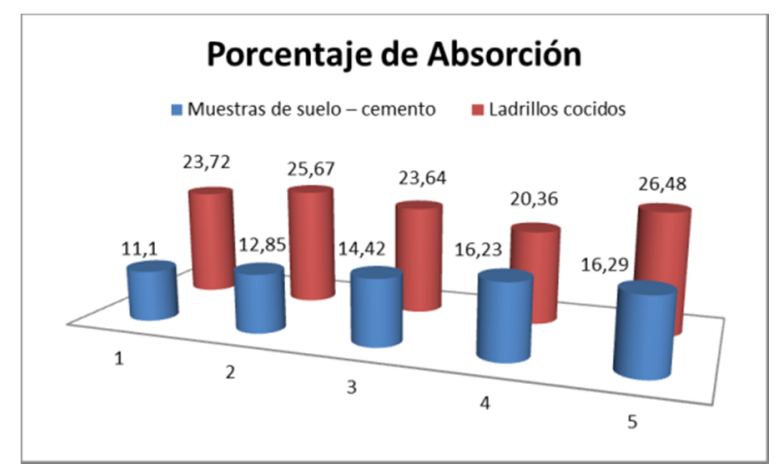

Fig. 7 Pruebas de porcentaje de absorción.

La implementación de la espectroscopia infrarroja ha sido un aporte adicional al desarrollo de este trabajo, a pesar de que este tipo de técnicas son más utilizadas para compuestos orgánicos también es muy útil para determinar cualitativamente los grupos funcionales presentes en una muestra con compuestos inorgánicos. El propósito de utilizar este tipo de técnica es corroborar por medio de los espectros de cada una de las muestras, los picos característicos de compuestos formados después de las reacciones de hidratación del cemento. Si comparamos los espectros de dos muestras una con cemento Canal y otra con Holcim, se puede apreciar la influencia que tiene utilizar dos tipos de marcas de cemento diferentes ya que sus composiciones son variables.

En la Fig. 8 se observa que poseen los mismos picos característicos de los compuestos, sin embargo poseen diferencias notables en relación al porcentaje de transmitancia que tienen los picos entre ambos espectros. La escala de la transmitancia entre ambos espectros es muy desigual y la diferencia más notable se encuentra en el pico del intervalo de 970 a $1100 \mathrm{~cm}^{-1}$ ya que para cemento Canal este llega a 
aproximadamente $68 \%$ y con cemento Holcim se alcanza cerca del $83 \%$. Lo anterior indica que hay una mayor cantidad de ese compuesto presente en el espectro.
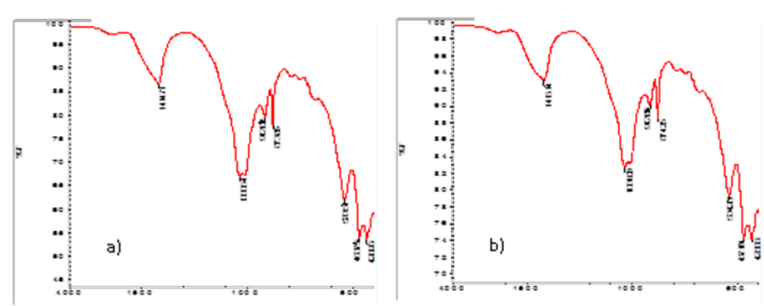

Fig. 8 Espectros de muestras con cemento Canal y Holcim. a) Canal y b) Holcim.

\section{Conclusiones}

Los resultados de las pruebas de RMC a los 28 días mostraron incrementos significativos para relaciones bajas de cemento/suelo de $1 / 4$ y relaciones altas de agua/ligante de 0.2 alcanzando resistencias superiores a los $12 \mathrm{MPa}$ en relación con ensayos que se realizaron para ladrillos cocidos que obtuvieron como promedio 9.35 MPa en forma de ladrillos completos y 3.11 $\mathrm{MPa}$ cortados en cubos. Los porcentajes promedio de absorción más altos fueron de los ladrillos cocidos alcanzando el $23.97 \%$, lo cual se debe a la cantidad de poros presentes en su estructura amorfa y a la falta de compactación de la mezcla que se elabora artesanalmente, por otro lado las muestras de suelo-cemento con relaciones de cemento/suelo de $1 / 4$ y 0.2 de agua/ligante tuvieron un porcentaje promedio de $14.17 \%$ mejorando de forma significativa las propiedades mecánicas de este. El uso de dos marcas de cemento de tipo Portland mostró el efecto que tiene sobre las propiedades mecánicas del material como es la RCM, ya que los valores máximos alcanzados se obtuvieron al utilizar el cemento canal superando los $12.57 \mathrm{MPa}$ a diferencia de las muestras con cemento Holcim que alcanzaron un máximo de resistencia de $9.80 \mathrm{MPa}$.

\section{Agradecimientos}

Los autores agradecemos a la ladrillería Bella Vista por haber abierto sus puertas ya que ha sido un elemento fundamental para éste trabajo de investigación. A la Facultad de Ingeniería Química (FIQ) y Facultad de Tecnología de la Construcción (FTC) por las gestiones realizadas para el desarrollo experimental de esta tesis. Finalmente al Laboratorio de Materiales y Modelos Estructurales de la Universidad de Costa Rica (LanammeUCR) por haber colaborado en los ensayos de espectroscopia infrarroja.

\section{Notación}

\begin{tabular}{|c|c|c|}
\hline Símbolo & Cantidad & Unidades \\
\hline $\mathrm{w}$ & $\begin{array}{l}\text { Fuerza ejercida } \\
\text { sobre la pieza }\end{array}$ & lbf \\
\hline A & $\begin{array}{l}\text { Área superficial } \\
\text { de la muestra }\end{array}$ & in $^{2}$ \\
\hline $\mathrm{C}$ & $\begin{array}{l}\text { Resistencia } \\
\text { mecánica a la } \\
\text { compresión }\end{array}$ & lbf/in ${ }^{2}$ \\
\hline W & $\begin{array}{l}\text { Peso de la } \\
\text { muestra }\end{array}$ & \\
\hline
\end{tabular}

\section{Subíndices}

$\begin{array}{ll}\text { s } & \text { Muestra } \\ & \text { saturada } \\ \text { d } & \text { Muestra seca }\end{array}$

\section{Referencias}

Amaya, M.A., Díaz, C.E. (2011). Manual de guías de laboratorio enfocado al control de calidad de materiales para las asignaturas: "Ingeniería de Materiales" y "Tecnología del Concreto". Tesis de Grado. Universidad de El Salvador, El Salvador.

Besoain, E. (1985). Mineralogía de arcillas de suelos. San José. Costa Rica. pp. 9-25.

Gatani, M. (2000) Ladrillos de suelo-cemento: mampuesto tradicional en base a un material sostenible. Arquitecta, CONICET, Vol. 51. Argentina. 